\title{
Ocular Larva Migrans: A Severe Manifestation of an Unseen Epidemic
}

\author{
Anna Quinn Hare • Carlos Franco-Paredes
}

Published online: 7 December 2013

(C) Springer International Publishing AG 2013

\begin{abstract}
Toxocara canis and Toxocara cati, which infect dogs and cats respectively, may cause infection in humans with a wide spectrum of clinical symptoms and morbidity. Prevalence studies have suggested more widespread affliction than indicated by level of awareness among clinicians in endemic areas. Transmission in the United States continues with seroprevalence estimates identified as high as $22 \%$ in some low-income communities. Best known for causing hepatitis and pneumonitis associated with the syndrome called visceral larva migrans, toxocariasis can also cause debilitating vision loss via ocular larva migrans and is considered a cause of preventable vision loss. While not a common manifestation of toxocariasis, the severity of this condition and its predilection for affecting children warrants increased attention. Herein, we review current knowledge of ocular larva migrans epidemiology, presentation, diagnosis, and treatment.
\end{abstract}

Keywords Ocular larva migrans $\cdot$ Toxocara canis $\cdot$ Toxocara cati - Toxocariasis · Epidemiology · Diagnosis · Treatment . Parasitology $\cdot$ Tropical medicine $\cdot$ Blindness $\cdot$ Retinal detachment $\cdot$ Parasitic infections

\section{Introduction}

The roundworm Toxocara spp, found in dogs and cats, has severe clinical manifestations in human infection, causing symptoms ranging from chronic cough to hepatitis to seizures

\author{
A. Q. Hare \\ Emory University School of Medicine, 80 Butler Street, Atlanta, \\ GA 30033, USA \\ e-mail: ahare@emory.edu \\ C. Franco-Paredes \\ Hospital Infantil de México, Federico Gómez, Mexico City, México \\ C. Franco-Paredes $(\triangle)$ \\ Phoebe Putney Memorial Hospital, Albany, GA, USA \\ e-mail: cfranco@ppmh.org
}

$[1,2]$. One particularly serious manifestation is debilitating vision loss, the result of ocular larva migrans (OLM). While not a common manifestation of toxocariasis $[3 \cdot, 4,5]$, both the severity of ramifications of OLM and the potential underreporting, based on seroprevalence studies, make OLM worthy of additional public attention $[5,6]$.

\section{Toxocariasis in Humans}

Dogs and cats shed unembryonated eggs in feces as part of the Toxocara spp lifecycle. Eggs take around 3 weeks to embryonate and become infective, after which they can exist for several years in soil [7]. When ingested by humans, or another non-host animal, the eggs hatch and the larvae penetrate the intestinal wall to reach the circulatory system and migrate throughout the body. The Toxocara larvae release an excretory-secretory antigen (TES) with high immunogenicity and allergenicity; this is the main stimulator of the human immune response [8].

Humans, typically children, acquire the infection via ingestion of eggs, likely in feces-contaminated soil, but potentially also via unwashed hands or vegetables. Dog ownership and pica (geophagia) have been highly associated with Toxocara infection $[7,9]$. T. canis has an estimated prevalence of $5 \%$ in dogs in the United States based on fecal examination in 44 states [10], and much higher in puppies [11]. Samples of soil taken from parks, playgrounds, and public areas in several locations in North America have shown high rates of soil contamination [7].

Despite some of the limitations in terms of specificity and sensitivity of currently available serological immunodiagnostic assays to indirectly measure human Toxocara spp infection, there is some consensus that there is underdiagnosis and under-reporting of this condition. Serological surveys performed in the U.S. in the early 70 s demonstrated higher infection rates among socioeconomically disadvantaged black and Hispanic children [2]. Recent seroprevalence studies 
among humans conducted as part of the National Health and Nutrition Examination Survey (NHANES) confirmed previous estimates by identifying Toxocara antibodies present nationally in $13.9 \%$ of individuals $\geq 6$ years old. Prevalence varied considerably by population group. The survey demonstrated $>22 \%$ prevalence among those living below the poverty line (based on the Department of Health and Human Services Poverty guidelines published yearly), more than $21 \%$ prevalence in non-Hispanic blacks, and $17.4 \%$ prevalence in the American Deep South [12]. While these measures of seroprevalence do not equate to either current infection or presence of severe symptoms $[8,11]$, they provide evidence of a higher infection prevalence than is indicated by the level of awareness of Toxocara infection and demonstrate that toxocariasis is most profound in areas and communities of poverty [13]. The prevalence of human toxocariasis in tropical settings is not well known, but it is likely that it is also underreported; its clinical manifestations, leading to severe morbidity and long-term sequelae, place this parasitic infection in the cateogry of a neglected tropical disease.

Humans are paratenic hosts, meaning not true hosts, to the Toxocara worm, and their lifecycle is not completed in humans. When Toxocara eggs are ingested by a human, the eggs hatch in the intestine and the larvae reach the circulatory system, but do not further develop. Instead, they migrate throughout the body and eventually become dormant and encased in tissue. The human host inflammatory immune response, activated by migrating larvae, their active release of TES, and the passive release upon their death, cause the majority of damage to tissues in organs reached by the larvae from the circulatory system [11]. The human physiologic response to infection is mediated primarily by a hypersensitivity response, which can be either immediate or delayedtype. The degree and type of clinical disease manifestations depends on several factors including the strength of the host immune response, the organs invaded, the degree of infection or larva burden, and the frequency of re-infection $[2,14]$.

There are three main clinical conditions recognized as being caused by Toxocara infection: visceral larva migrans (VLM), covert toxocariasis, and ocular larva migrans (OLM) that may occur among socioeconomically disadvantaged populations. These syndromes very rarely occur together, as they represent different degrees and locations of infestation and immune response [15]. VLM occurs with a heavy larva burden and invasion of sensitive organs, namely liver, lungs, and central nervous system, typically manifesting as hepatitis, pneumonitis or seizures if larvae penetrate the central nervous system $[11,16 \bullet \bullet]$. VLM mainly affects young children averaging 6 months to 3 years of age [2]. Covert toxocariasis has relatively unknown prevalence due to its nebulous symptoms such as headache, abdominal pain, cough, fever, and wheezing, but seems to span a wide range of ages and has been linked using serology to decreased lung function over time [12,
$16 \bullet \bullet, 17,18]$. OLM is the least common manifestation of Toxocara infection, is more typical of older children and teenagers, and occurs when even a single larva enters the eye. OLM may cause significant visual disability and blindness $[8,9,19,20 \bullet \bullet, 21]$.

\section{Epidemiology of Ocular Toxocara infection}

Ocular larva migrans, sometimes referred to as ocular toxocariasis, is an uncommon manifestation of infection with Toxocara spp. in humans. Prevalence of OLM is difficult to estimate due to a combination of lack of adequate studies, poor association between clinical OLM disease and serologic prevalence of Toxocara antibodies, and lack of awareness of OLM. There have been a few geographically limited prevalence studies in the U.S. over the past 30 years [5, 21], but the largest and most recent indication of prevalence of OLM comes from a national survey of ophthalmologists conducted by the Centers for Disease Control (CDC) in collaboration with the American Academy of Ophthalmology. Results of this survey found 68 reported cases of OLM in the U.S. from September 2009 to September 2010. This survey was limited by low participation (19\%), as well as the likelihood of both under-reporting of less severe clinical disease by providers and higher prevalence of toxocariasis in areas of poverty with less access to healthcare, where cases may go undocumented and untreated. Nevertheless, this important survey provides a starting estimate for assessing the prevalence of ocular toxocariasis by population and location in the U.S. (Table 1). This study demonstrates a significantly higher prevalence of OLM in specific areas. From 2009-2010, there were more cases of OLM reported in the southern states compared to all other regions combined, with the State of Georgia having the highest number of cases. The survey also demonstrates a higher prevalence within urban and suburban communities. Given study limitations noted above and paucity of additional large-scale studies of OLM prevalence, associations between Toxocara seroprevalence and prevalence of OLM cannot be concretely determined.

\section{Mechanism of Disease in Ocular Larva Migrans}

The unique and subtle presentation of OLM suggests a mechanism of acquiring ocular Toxocara infection. Because it typically does not present with other symptoms of toxocariasis and patients are otherwise completely asymptomatic and without elevated WBC or eosinophilia, it is likely that the larva burden is low $[8,15]$. With a very low larva burden, the immune system is not sufficiently activated to isolate or disable the larva and it has time to migrate throughout the body until it enters the eye. Those larvae that do not continue to develop into adults can have a lifespan of several years in the human 
Table 1 Number and percentage of patients with newly diagnosed OLM by selected characteristics - United States 2009-2010 (N=68)

\begin{tabular}{|c|c|c|}
\hline Demographic & No of patients & $\%$ \\
\hline \multicolumn{3}{|l|}{ Age (yrs) $(n=44)$} \\
\hline Median & 8.5 & \\
\hline Range & $1-60$ & \\
\hline \multicolumn{3}{|l|}{$\operatorname{Sex}(n=43)$} \\
\hline Male & 24 & 56 \\
\hline Female & 19 & 44 \\
\hline \multicolumn{3}{|l|}{ Race $(n=43)$} \\
\hline White & 30 & 70 \\
\hline Black & 8 & 19 \\
\hline Asian/Pacific Islander & 4 & 9 \\
\hline American Indian & 1 & 2 \\
\hline \multicolumn{3}{|l|}{ Ethnicity $(\mathrm{n}=41)$} \\
\hline Hispanic & 7 & 17 \\
\hline Non-Hispanic & 34 & 83 \\
\hline \multicolumn{3}{|l|}{ Region $(n=44)$} \\
\hline Northeast & 6 & 14 \\
\hline Midwest & 5 & 11 \\
\hline South & 25 & 57 \\
\hline West & 8 & 18 \\
\hline \multicolumn{3}{|l|}{ Environment $(\mathrm{n}=40)$} \\
\hline Urban & 12 & 30 \\
\hline Rural & 7 & 18 \\
\hline Suburban & 16 & 40 \\
\hline Mixed & 5 & 12 \\
\hline Pet ownership ( $\mathrm{n}=33$ ) & 21 & 64 \\
\hline \multicolumn{3}{|l|}{ Type of pet $(n=21)$} \\
\hline Dog & 9 & 43 \\
\hline Cat & 6 & 29 \\
\hline Dog and cat & 5 & 24 \\
\hline Unknown & 1 & 5 \\
\hline \multicolumn{3}{|c|}{ Health insurance coverage $(n=43)$} \\
\hline Private & 18 & 42 \\
\hline Medicare & 16 & 37 \\
\hline Medicaid & 0 & \\
\hline Uninsured & 3 & 8 \\
\hline Unknown & 6 & 14 \\
\hline
\end{tabular}

(Taken from Reference 20: Lum FC, Hoskins HD, Moorthy RS, Read RW, Starr MC, Jones JL, et al. Ocular Toxocariasis - United States, 20092010. MMWR 2011,60:734-736)

body [2]. It is possible that children with OLM have had a very small number of larvae in their systems for a longer period of time, for example due to repeated small-dose inoculation [22]. The small larva burden would fail to incite immune response while providing more time for chance ocular migration and older age on presentation. The unilateral nature and the fact that OLM typically occurs in children slightly older than those typically afflicted with VLM support this concept.
Ocular larva migrans is typically caused by migration of Toxocara spp larvae through the choroidal and retinal vessels into the posterior segment of the eye, or occasionally through the optic nerve via the central nervous system [23, 24]. There is no evidence in the literature that anything particularly draws Toxocara larvae to the eye in humans. Models of OLM in monkeys have attempted to discover whether there is a predilection of the larvae for the eye, but at this point, inoculation in any location besides the brain and the eye itself yields rare and unpredictable intraocular infection. Models inoculating the carotid artery, the stomach, periocular tissue, and systemic inoculation have all shown rare ocular infection [22]. Mongolian gerbil models seem to demonstrate some predilection for the eye, given the high prevalence of ocular lesions after infection with low larva burden [25], but this mechanism is yet unknown and has not been demonstrated in either humans or other animal models.

Even with intraocular injection, considerable variation exists in response to ocular presence of Toxocara larvae. One model using cynomolgus monkeys with intravitreal injection of viable, embrionated Toxocara larvae demonstrated several interesting findings supporting this variability [22]. First, not all intraocular larvae incited an immune response, and viable larvae were found in the vitreous, retina, and optic nerve up to 9 months after inoculation, without inflammatory response. This demonstrates large variability in the immune response to intraocular larvae. It remains unclear whether this variation is due to differences in larval activity and toxin release or specific immune characteristics of the host. Second, while several larvae did incite immune response in the form of acute inflammatory granuloma or chronic fibrotic granuloma, the surrounded larvae did not appear to be necrotic, and several larvae demonstrated ability to move through ocular tissue and leave the site of reaction. Third, when examined 3 days after inoculation, no reaction was seen in any of the eyes, showing that brief occupation of the eye may not incite immune response. Finally, while dead larvae caused little reaction, Toxocara proteins intravitreally injected stimulated severe retinal vasculitis. These results, though just a model of infection in humans, suggest considerable variability in response to intraocular infection by Toxocara larvae.

\section{Clinical Findings in Ocular Larva Migrans}

OLM typically occurs as the sole manifestation of toxocariasis in individuals with no antecedent history symptoms, though may rarely be accompanied by symptoms of covert toxocariasis, such as wheezing, or symptoms of VLM [9, 11]. Typical presentation of OLM is unilateral in the majority of cases and most commonly affects older children than those afflicted by VLM $[3 \cdot, 4,9,11]$. Most cases of OLM are otherwise asymptomatic, have a normal WBC and no eosinophilia, and can have negative serology $[2,4,8,15]$. 
The most common presenting clinical findings include strabismus, diminished vision, photophobia, leukocoria, vitritits, ocular injection, and pain (Table 2) [3•, 4, 11, 20••, 21]. Based on one study of patient data spanning 19 years, presenting vision loss from OLM was most commonly associated with vitritis, cystoid macular edema, and traction retinal macular detachment [21]. Though the larva is not known to invade the optic nerve, there are reports of atrophy of the optic nerve head due to intraocular larvae [11], which based on animal models are thought to enter via the central nervous system rather than retinal vessels [24]. The degree of disability from OLM varies. Patients can present with vision loss ranging from 20/40 to hand movements [3•]. Infection may also be subclinical and only diagnosed on routine eye exam [9].

\section{Diagnosis, Treatment and Outcome of Ocular Larva Migrans}

Due to the typically low larva burden, OLM is difficult to diagnosis, and many cases remain presumed toxocariasis

Table 2 Number and percentage of patients with newly diagnosed OLM by signs and symptoms present at examination - United States 2009$2010(\mathrm{~N}=68)$

\begin{tabular}{lll}
\hline Clinical Feature & Number & $\%$ \\
\hline Signs (n=32) & & \\
Subretinal granulomatous mass/scar Vitritis & 20 & 65 \\
Vitritis & 16 & 55 \\
Scotoma & 10 & 50 \\
Posterior pole granuloma & 13 & 42 \\
Peripheral granuloma with traction bands & 12 & 39 \\
Active chorioretinitis & 11 & 34 \\
Retinal detachment & 9 & 28 \\
Strabismus & 8 & 27 \\
Anterior uveitis & 8 & 25 \\
Leukocoria & 4 & 15 \\
Diffuse nematode endophthalmitis & 2 & 6 \\
Symptoms (n=37) & & \\
Vision loss & 25 & 83 \\
Permanent & 17 & 68 \\
Temporary & 7 & 28 \\
Unknown & 1 & $<1$ \\
Floaters & 13 & 38 \\
Eye redness & 12 & 32 \\
Photophobia & 10 & 27 \\
Eye pain & 7 & 19 \\
\hline
\end{tabular}

(Taken from Reference 20: Lum FC, Hoskins HD, Moorthy RS, Read RW, Starr MC, Jones JL, et al. Ocular Toxocariasis - United States, 2009 2010. MMWR 2011,60:734-736) without validating serology or visualization of larvae [9, 14]. Ophthalmic examination and identification of typical features is important in the diagnosis of OLM [2]. Diagnosis therefore depends on a combination of fundoscopy, serology, and histopathologic identification of larvae of surgically obtained specimens.

The mainstay of medical treatment is a combination of glucocorticoids, antihelminthic therapy; many cases require vitrectomy depending on the severity and mechanism of damage. Because intra-ocular inflammation is the main cause of damage and visual impairment via inflammation-induced tissue injury and secondary membrane formation, treatment for OLM focuses primarily on controlling inflammation with steroids. Topical or periocular corticosteroids are used. Prednisone may be added at $0.5-1 \mathrm{mg} / \mathrm{kg} /$ day for systemic steroid treatment in cases of severe inflammation [14, 15, 26]. Antihelminthic treatment can also be used in OLM, particularly with concomitant extra-ocular toxocariasis symptoms. Recommended antihelminthic agents include diethylcarbamazine and albendazole; the latter crosses the blood-brain barrier and has shown potential for larvacidal activity in host tissues $[15,27]$. Surgery, laser, or cryotherapy is considered for some cases of OLM. Treatment with an antihelminthic agent and high dose steroids has shown to be effective treatment in cases with Toxocara-associated uveitis with no remissions, but return of full vision does not appear common from literature, most likely due to structural changes[15, 26]. Outcome and residual visual deficits after treatment for OLM thus vary considerably, but full return of vision is unlikely and visual improvement is not guaranteed with any treatment modality.

\section{Conclusion}

Ocular Larva Migrans is a severely debilitating manifestation of Toxocara infection that, though rare, is difficult to prevent, diagnose, and treat. While new technologies or techniques for diagnosis and treatment are needed to improve outcome in late-detected infections, the largest gains in combating OLM will come from increased public and medical community awareness of the condition in the U.S. and also in other settings. Better public awareness could also increase parasite control in pets and animals nationally. Increased public and parental awareness could help reduce the amount of larvae in environments most utilized by children, such as playgrounds, via improved pet regulations, monitoring of children, or treatment of soil/sand in those areas. And most importantly, better awareness among physicians, pediatricians, optometrists, and ophthalmologists could result in earlier diagnosis of ocular manifestations of Toxocara in children, leading to earlier treatment and prevention of severe vision loss. 


\section{Compliance with Ethics Guidelines}

Conflict of Interest Anna Quinn Hare and Carlos Franco-Paredes declare that they have no conflict of interest.

Human and Animal Rights and Informed Consent This article does not contain any studies with human or animal subjects performed by any of the authors.

\section{References}

Papers of particular interest, published recently, have been highlighted as:

- Of importance

- Of major importance

1. Gavignet B, Piarroux R, Aubin F, Millon L, Humbert P. Cutaneous manifestations of human toxocariasis. J Am Acad Dermatol. 2008;59:1031-42.

2. Despommier D. Toxocariasis: clinical aspects, epidemiology, medical ecology, and molecular aspects. Clin Microbiol Rev. 2003;16:265-72.

3. Schneier AJ, Durand ML. Ocular toxocariasis: advances in diagnosis and treatment. Int Ophthalmol Clin. 2011;51:135-44. This report provides a comprehensive review of clinical issues of ocular toxocariasis.

4. Taylor MR. The epidemiology of ocular toxocariasis. J Helminthol. 2001;75:109-18.

5. Maetz HM, Kleinstein RN, Federico D, Wayne J. Estimated Prevalence of Ocular Toxoplasmosis and Toxocariasis in Alabama. J Infect Dis. 1987;156:414.

6. Sharghi N, Schantz PM, Caramico L, Ballas K, Teague BA, Hotez PJ. Environmental exposure to Toxocara as a possible risk factor for asthma: a clinic-based case-control study. Clin Infect Dis. 2001;32: E111-116.

7. Glickman LT, Schantz PM. Epidemiology and Pathogenesis of Zoonotic Toxocariasis. Epidemiol Rev. 1981;3:230-50.

8. Fillaux J, Magnaval JF. Laboratory diagnosis of human toxocariasis. Vet Parasitol. 2013;193:327-36.

9. Good B, Holland CV, Taylor MRH, Larragy J, Moriarty P, O'Regan M. Ocular Toxocariasis in Schoolchildren. Clin Infect Dis. 2004;39:173-8.

10. Mohamed AS, Moore GE, Glickman LT. Prevalence of intestinal nematode parasitism among pet dogs in the United States (20032006). J Am Vet Med Assoc. 2009;234:631-7.

11. Shields JA. Ocular toxocariasis a review. Surv Ophthalmol. 1984;28:361-81.

12. Won KY, Kruszon-Moran D, Schantz PM, Jones JL. National seroprevalence and risk factors for zoonotic toxocara spp. infection. Am J Trop Med Hyg. 2008;79:552-7.
13. Hotez PJ. Neglected Infections of Poverty in the United States of America. PLoS Negl Trop Dis. 2008;2:e256.

14. Arevalo JF, Espinoza JV. Retinal and Choroidal Manifestations of Selected Systemic Diseases. In: Retnal and Chrroidal Manifestations of Selected Systemic Diseases. Edited by Arevalo JF. 17 ed: Arevalo, J. F. ; 2013. pp. 47-62.

15. Barisani-Asenbauer T, Maca SM, Hauff W, Kaminski SL, Domanovits H, Theyer I, et al. Treatment of ocular toxocariasis with albendazole. J Ocul Pharmacol Ther. 2001;17:287-94.

16.• Hotez PJ, Wilkins PP. Toxocariasis: America's most common neglected infection of poverty and a helminthiasis of global importance? PLoS Negl Trop Dis. 2009;3:e400. This editorial summarizes the epidemiology of what is known about toxocariasis in the United States and presents a detailed discussion of research agenda of this neglected disease of poverty.

17. Taylor MR, Keane CT, O'Connor P, Girdwood RW, Smith H. Clinical features of covert toxocariasis. Scand J Infect Dis. 1987;19:693-6.

18. Walsh MG. Toxocara infection and diminished lung function in a nationally representative sample from the United States population. Int J Parasitol. 2011;41:243-7.

19. Woodhall D, Starr MC, Montgomery SP, Jones JL, Lum F, Read RW, et al. Ocular toxocariasis: epidemiologic, anatomic, and therapeutic variations based on a survey of ophthalmic subspecialists. Ophthalmology. 2012;119:1211-7.

20.• Lum FC, Hoskins HD, Moorthy RS, Read RW, Starr MC, Jones JL, et al. Ocular Toxocariasis - United States, 2009-2010. MMWR. 2011;60:734-6. This report is colaboration of the CDC and the American Academy of Ophtalmology that demonstrates that ocular toxocariasis is a preventable zoonotic parasitic infections that may cause major vision loss primarily in children.

21. Stewart JM, Cubillan LDPCE. Prevalence, clinical features, and causes of vision loss among patients with ocular toxocariasis. Retina. 2005;25:1005-13.

22. Watzke RC, Oaks JA, Folk JC. Toxocara canis infection of the eye. Correlation of clinical observations with developing pathology in the primate model. Arch Ophthalmol. 1984;102: 282-91.

23. Kim YJ, Moon CH, Chang JH. Toxocariasis of the optic disc. J Neuroophthalmol. 2013;33:151-2.

24. Hayashi E, Akao N, Fujita K. Evidence for the involvement of the optic nerve as a migration route for larvae in ocular toxocariasis of Mongolian gerbils. J Helminthol. 2003;77:311-5.

25. Zanandrea LI, Oliveira GM, Abreu AS, Pereira FE. Ocular lesions in gerbils (Meriones unguiculatus) infected with low larval burden of Toxocara canis: observations using indirect binocular ophthalmoscopy. Rev Soc Bras Med Trop. 2008;41:570-4.

26. Dinning WJ, Gillespie SH, Cooling RJ, Maizels RM. Toxocariasis: a practical approach to management of ocular disease. Eye (Lond). 1988;2(Pt 5):580-2.

27. Magnaval JF, Glickman LT, Dorchies P, Morassin B. Highlights of human toxocariasis. Korean J Parasitol. 2001;39:1-11. 